\title{
Inducing the cosmological constant from five-dimensional Weyl space
}

\author{
José Edgar Madriz Aguilar, and Carlos Romero \\ ${ }^{a}$ Departamento de Física, Universidade Federal da Paraíba, \\ Caixa Postal 5008, 58059-970 \\ João Pessoa, PB, Brazil \\ E-mail: jemadriz@fisica.ufpb.br; cromero@fisica.ufpb.br
}

\begin{abstract}
We investigate the possibility of inducing the cosmological constant from extra dimensions by embedding our four-dimensional Riemannian space-time into a five-dimensional Weyl integrable space. Following approach of the induced matter theory we show that when we go down from five to four dimensions, the Weyl field may contribute both to the induced energy-tensor as well as to the cosmological constant $\Lambda$, or more generally, it may generate a time-dependent cosmological parameter $\Lambda(t)$. As an application, we construct a simple cosmological model in which $\Lambda(t)$ has some interesting properties.
\end{abstract}

PACS numbers: 04.20.Jb, 11.10.kk, 98.80.Cq

Keywords: Five-dimensional vacuum, Integrable Weyl theory of gravity, Induced-matter theory

\section{INTRODUCTION}

In a very recent past it appeared that the role played by the cosmological constant in Cosmology was merely historical, mainly connected with Einstein's attempt to build a cosmological scenario in which the Universe was static and finite [1] . However, since the recent discovery of cosmic acceleration there has been a renewed interest in the role the cosmological constant could play to explain the new data. For instance, there is strong evidence that the so-called dark energy, which would cause the Universe's accelerated expansion, might have a connection with the cosmological constant. Moreover, the present most popular model of cosmology, the Lambda-CDM model, tacitally assumes the existence of the cosmological constant [2]. On the other hand, particle physics theorists have always argued if favour of the existence of the cosmological constant as a consequence of the energy density of the vacuum [ 3 ].

From the standpoint of cosmological theory it seems then desirable to have a justification of the cosmological constant on theoretical grounds. This quest has led some theoreticians to modify Einstein's gravitational theory, these attempts going back to the works of Eddington and Schrödinger [4].

Our aim in the present article is to introduce a new approach to this old question. We argue that the appearance of the cosmological constant in the equations of four-dimensional (4D) general relativity might be related to the assumption, made by some modern spacetime theories, that our Universe may have extra dimensions. Among such theories are the so-called braneworld scenario [5] and the non-compact Kaluza-Klein theories [6], both sharing some basic assumptions concerning the geometry of the fundamental higher-dimensional embedding space. For example, in these proposals our ordinary spacetime is viewed as a hypersurface (the brane) embedded in a five-dimensional (5D) manifold (the bulk). On the other hand, mathematical theorems regulate these embeddings, in particular, the Campbell-Magaard theorem [7] and its extensions specify the conditions under which the embeddings are possible [8].

The possibility of generating matter and fields from a higher-dimensional vacuum was first realized by T. Kaluza and O. Klein, an idea that has been a source of inspiration for practically all higher-dimensional theories [9]. In particular, a mathematical formalism has been developed by Wesson and Ponce de Leon 10] that permits to "induce" an energy-momentum tensor from the vacuum Einstein field equations in five dimensions, a schema now known as the induced-matter proposal. It has later been shown that from a geometrical point of view such generated energymomentum tensor is nothing more than the extrinsic curvature of the spacetime hypersurface in disguise [11] . Besides, an interesting feature of this mechanism, apart from this "geometrization" of matter, is that it is also powerful enough to generate a cosmological constant in four dimensions out of a pure five-dimensional vacuum [12]. It should be noted, however, that in the context of the usual induced matter approach by considering a Riemannian bulk it has not been possible until now to generate simultaneously the cosmological constant and an induced energy-momentum tensor $T_{\alpha \beta}^{(I M)}$ that describes macroscopic matter, at least without modifying the "canonical" form of $T_{\alpha \beta}^{(I M)}[\underline{6}]$. The interesting point here is that this apparent weakness can be remedied if we allow for the geometry of the embedding space having more degrees of freedom such as in the case of Weyl geometry [13]. It turns out then that when we go down from $5 \mathrm{D}$ to $4 \mathrm{D}$, as in the induced matter approach, the Weyl field may contribute both to the induced energytensor as well as to the cosmological constant $\Lambda$, or more generally, to a time-dependent cosmological parameter 
$\Lambda(t)$

In this paper we shall consider a particular case of Weyl geometry, namely, the one in which the Weyl field is integrable 14]. It turns out that in this case if the Weyl field depends only on the extra dimension, then the embedded spacetime is Riemannian and general relativity holds in the brane [15], although the non-Riemannian character of the whole bulk propagates into the brane in the form of induced matter and a cosmological parameter.

The paper is organized as follows. We start in Section I with a brief review of some fundamental concepts that underlie Weyl geometry. We proceed in Section II to develop a five-dimensional Weylian theory of gravity in vacuum, the dynamics of which is given by a certain action chosen among the simplest ones [16]. In the same section we set the equations for a particular choice of the five-dimensional metric and examine the case of a simple cosmological model. In Section III we illustrate the theory by going through a simple example taken from Cosmology. Our final remarks are contained in Section IV.

\section{WEYL GEOMETRY}

Conceived by H. Weyl in 1918, as an attempt to unify gravity with electromagnetism, in its original form Weyl's theory [13] turned out to be innadequate as a physical theory as was firstly pointed by Einstein soon after the appearance of the theory [14]. However, a variant of Weyl geometry, known as Weyl integrable geometry does not suffer from the drawback pointed out by Einstein and for this reason has attracted the attention of some cosmologists [16], particularly in the context of higher-dimensional theories [17].

The starting point of the new geometry created by Weyl is the assumption that the covariant derivative of the metric tensor $g$ is not zero, but, instead, is given by

$$
\nabla_{a} g_{b c}=\sigma_{a} g_{b c}
$$

where $\sigma_{a}$ denotes the components of a one-form field $\sigma$ with respect to a local coordinate basis. This is, of course, a generalization of the idea of Riemannian compatibility between the connection $\nabla$ and $g$, which is equivalent to require the length of a vector to remain unaltered by parallel transport [14]. If $\sigma=d \phi$, where $\phi$ is a scalar field, then we have an integrable Weyl geometry. A differentiable manifold $M$ endowed with a metric $g$ and a Weyl field $\sigma$ is usually referred to as a Weyl frame. It is interesting to see that the Weyl condition (11) remains unchanged when we go to another Weyl frame $(M, \bar{g}, \bar{\sigma})$ by performing the following simultaneous transformations in $g$ and $\sigma$ :

$$
\begin{gathered}
\bar{g}=e^{-f} g \\
\bar{\sigma}=\sigma-d f
\end{gathered}
$$

where $f$ is a scalar function defined on $M$.

Our next step is to consider a Weylian theory of gravity. If we consider a Weyl spacetime the simplest action that gives the dynamics of the gravitational field in the absence of matter is given by

$$
\mathcal{S}=\int d^{4} x \sqrt{g}\left[\mathcal{R}+\xi \phi_{; a}^{a}\right]
$$

where $\xi$ is an arbitrary coupling constant, $\phi_{a} \equiv \phi_{, a}$ is the the Weyl field, $\mathcal{R}$ is the Weylian Ricci scalar and the semicolon (;) denotes covariant derivative with respect to the Weyl connection [16]. The extension of this formulation to a higher-dimensional space is straightforward. In the next section we shall consider the a five-dimensional Weyl integrable space.

\section{A WEYL INTEGRABLE DYNAMICS IN FIVE DIMENSIONS}

Let us consider a five-dimensional space $M^{5}$ endowed with a metric tensor ${ }^{(5)} \mathrm{g}$ and an integrable Weyl scalar field $\phi$. In local coordinates $\left\{y^{a}\right\}$ the five-dimensional line element of will be denoted by

$$
d S^{2}=g_{a b}(y) d y^{a} d y^{b}
$$

where $g_{a b}$ are the components of ${ }^{(5)} \mathrm{g}$. As we already mentioned the simplest action that can be constructed for a Weylian theory of gravity in a five-dimensional vacuum is given by

$$
{ }^{(5)} \mathcal{S}=\int d^{5} y \sqrt{\left|{ }^{(5)} g\right|}\left[{ }^{(5)} \mathcal{R}+\xi \phi^{a} ; a\right],
$$


where $\xi$ is an arbitrary coupling constant, $\phi_{a} \equiv \phi_{, a}$ is the gauge vector associated to the Weyl field, $\left|{ }^{(5)} g\right|$ is the absolute value of the determinant of the metric ${ }^{(5)} g_{a b},{ }^{(5)} \mathcal{R}$ is the Weylian Ricci scalar. One can easily check that the variation of the action (6) with respect to the tensor metric and with respect to the Weyl scalar field yields

$$
\begin{gathered}
{ }^{(5)} \mathcal{G}_{a b}+\phi_{a ; b}-(2 \xi-1) \phi_{a} \phi_{b}+\xi g_{a b} \phi_{c} \phi^{c}=0 \\
\phi_{; a}^{a}+2 \phi_{a} \phi^{a}=0
\end{gathered}
$$

where ${ }^{(5)} \mathcal{G}_{a b}$ denotes the Einstein tensor calculated with the Weyl connection ${ }^{(5)} \Gamma_{b c}^{a}={ }^{(5)}\left\{\begin{array}{c}a \\ b c\end{array}\right\}-(1 / 2)\left[\phi_{b} \delta_{c}^{a}+\phi_{c} \delta_{b}^{a}-g_{b c} \phi^{a}\right]$ and $\left\{\begin{array}{c}a \\ b c\end{array}\right\}$ are the Christoffel symbols of Riemannian geometry. The equations (7) and (8) are the field equations of the five-dimensional Weyl gravitational theory and describes the dynamics of a five-dimensional bulk in vacuum. A better insight may be gained if we recast the field equations (7) and (8) into its Riemannian part plus the contribution of the Weyl scalar field. Thus after excluding total derivatives of the scalar field $\phi$ the action (6) can be written as [16]

$$
{ }^{(5)} \mathcal{S}=\int d^{5} y \sqrt{g_{5}}\left[{ }^{(5)} \tilde{R}+\frac{1}{2}(5 \xi-6) \phi_{a} \phi^{a}\right]
$$

The field equations are obtained by taking a variation of the above action with respect to the pair $\left(g_{a b}, \phi\right)$. We are then led to

$$
\begin{aligned}
& { }^{(5)} \tilde{G}_{a b}-\frac{1}{2}(6-5 \xi)\left[\phi_{a} \phi_{b}-\frac{1}{2} g_{a b} \phi_{c} \phi^{c}\right]=0, \\
& { }^{(5)} \tilde{\square} \phi=0,
\end{aligned}
$$

where the tilde $(\sim)$ is used to denote quantities calculated with the Riemannian part of the Weyl connection and (5) $\tilde{\square}$ denotes the 5D d'Alembertian operator in the Riemannian sense.

At this point let us express the local coordinates $\left\{y^{a}\right\}$ as $\left\{x^{\alpha}, l\right\}$ denoting by $l$ the fifth (spacelike) coordinate and we choose for simplicity the line element (5) in the form ${ }^{1}$

$$
d S^{2}=g_{\alpha \beta}(x, l) d x^{\alpha} d x^{\beta}-\Phi^{2}(x, l) d l^{2} .
$$

where the function $\Phi^{2}(x, l)$ is the 5D analogue of the lapse function used in canonical general relativity, which supposes that spacetime may be foliated by a family of spacelike surfaces [19].

As in the induced matter approach [10], with respect to this foliation the field equations (10) can be splitted as

$$
\begin{gathered}
{ }^{(5)} \tilde{G}_{\alpha \beta}-\frac{1}{2}(6-5 \xi)\left[\phi_{\alpha} \phi_{\beta}-\frac{1}{2} g_{\alpha \beta}\left(\phi_{\gamma} \phi^{\gamma}-\Phi^{-2} \phi_{l}^{2}\right)\right]=0, \\
{ }^{(5)} \tilde{G}_{\alpha l}-\frac{1}{2}(6-5 \xi) \phi_{\alpha} \phi_{l}=0, \\
{ }^{(5)} \tilde{G}_{l l}-\frac{1}{4}(6-5 \xi)\left[\phi_{l}^{2}+\Phi^{2} \phi_{\gamma} \phi^{\gamma}\right]=0 .
\end{gathered}
$$

The five-dimensional Weylian equations we have now obtained assuming the geometry given by (12) supposes that in principle the Weyl scalar field $\phi$ depends on all coordinates, that is, $\phi=\phi(x, l)$. Some solutions of the above field equations have been worked out in detail by Novello and colaborators considering different geometric settings [16]. However our interest here is mainly to study a particular case of these field equations when the four-dimensional spacetime can be embedded in a five-dimensional ambient space whose dynamics comes from an integrable Weyl theory of gravity. In fact this is strongly motivated by a recently result concerning the existence of necessary and sufficient conditions for a Riemannian manifold to be embedded in a Weyl space [15]. According to these, if the Weyl scalar

\footnotetext{
${ }^{1}$ We shall adopt the convention $\operatorname{diag}(+---)$ for the signature of $g_{\alpha \beta}$.
} 
field depends only on the extra coordinate $l$, then each leaf of the foliation $l=$ const has a Riemannian character and can be locally and isometrically embedded in a five-dimensional Weylian space whose metrical properties are given by (12). Since we regard the spacetime as one of the leaves of the foliation and given that such embedding preserves the Riemannian character of the spacetime we proceed to investigate the four-dimensional field dynamics induced by the five-dimensional space. In much the same way as in induced-matter theory [10] one would interpret the extra contributions coming from the extra dimension as macrosocopic matter in 4D.

In view of the above let us assume that $\phi=\phi(l)$, i.e the Weyl scalar field depends only on the extra coordinate $l$. In this case the field equations (13), (14) and (15) become

$$
\begin{aligned}
& { }^{(5)} \tilde{G}_{\alpha \beta}+\frac{1}{4}(5 \xi-6) \Phi^{-2} g_{\alpha \beta} \phi_{l}^{2}=0, \\
& { }^{(5)} \tilde{G}_{\alpha l}=0, \\
& { }^{(5)} \tilde{G}_{l l}-\frac{1}{4}(6-5 \xi) \phi_{l}^{2}=0, \\
& \frac{\partial}{\partial l}\left[\sqrt{\left|g_{5}\right|} \Phi^{-2} \phi_{l}^{2}\right]=0 .
\end{aligned}
$$

To illustrate with an example let the five-dimensional space $M^{5}$ correspond to a five-dimensional cosmological model in the form of a triple warped product manifold [18] with metric given by

$$
d S^{2}=d t^{2}-a^{2}(t) d r^{2}-e^{2 F(t)} d l^{2}
$$

where $d r^{2}=\delta_{i j} d x^{i} d x^{j}$ is the three-dimensional Euclidian line element, $t$ represents the cosmic time for co-moving observers, $F(t)$ is a well-behaved real function and $a(t)$ is the cosmological scale factor. Inserting the metric (20) in (19) it can be easily seen that the Weyl scalar field in this case is given by

$$
\phi(l)=C_{1} l+C_{2},
$$

where $C_{1}$ and $C_{2}$ are integration constants. The field equations (16), (17) and (18) now give

$$
\begin{aligned}
3 H^{2}+3 \dot{F} H & =\frac{1}{4}(6-5 \xi) C_{1}^{2} e^{-2 F}, \\
2 \frac{\ddot{a}}{a}+H^{2}+2 \dot{F} H+\ddot{F}+\dot{F}^{2} & =\frac{1}{4}(6-5 \xi) C_{1}^{2} e^{-2 F}, \\
3\left(\frac{\ddot{a}}{a}+H^{2}\right) & =-\frac{1}{4}(6-5 \xi) C_{1}^{2} e^{-2 F},
\end{aligned}
$$

where $H(t)=\dot{a} / a$ is the Hubble parameter. From (23) and (24) we obtain the equation

$$
\ddot{F}+\dot{F}^{2}+2 H \dot{F}+5 \frac{\ddot{a}}{a}+4 H^{2}=0 .
$$

In order to simplify the structure of this equation we introduce a new function $u(t)$ defined by $u(t)=a(t) e^{F(t)}$. In this way (25) becomes

$$
\ddot{u}+4\left(\frac{\ddot{a}}{a}+H^{2}\right) u=0 .
$$

The above equation relates $u(t)$ with $a(t)$ in such a way that the solutions of (26) can be substituted in (22) yielding a differential equation for $a(t)$, which in principle can be solved.

\section{THE DYNAMICS INDUCED ON THE FOUR-DIMENSIONAL RIEMANNIAN BRANE}

As we have mentioned in the previous section one of our aims is to explore the possibility of interpreting the extra contributions of the five-dimensional Weylian bulk to the four-dimensional Riemannian brane as four-dimensional matter induced geometrically. In this section we shall study the four-dimensional dynamics geometrically induced on a generic brane. We recalll that we are assuming that the five-dimensional space is foliated by a family of hypersurfaces 
$\{\Sigma\}$ defined by the equation $l=$ const. Clearly, on a particular hypersurface $\Sigma_{0}$ the induced line element will be given by

$$
d S_{\Sigma_{0}}^{2}=h_{\alpha \beta}(x) d x^{\alpha} d x^{\beta}
$$

where $h_{\alpha \beta}(x)=g_{\alpha \beta}\left(x, l_{0}\right)$ is the induced metric on $\Sigma_{0}$. From the Gauss-Codazzi equations it is easy to show (See, for instance [17]) that the induced dynamics on the hypersurface $\Sigma_{0}$ is governed by the four-dimensional field equations

$$
{ }^{(4)} \tilde{G}_{\alpha \beta}=T_{\alpha \beta}^{(I M)}+\Lambda(x) h_{\alpha \beta},
$$

where $T_{\alpha \beta}^{(I M)}$ is the usual energy momentum tensor obtained in the induced matter approach, which has the form [10]

$$
T_{\alpha \beta}^{(I M)}=\frac{\Phi_{\alpha \| \beta}}{\Phi}+\frac{1}{2 \Phi^{2}}\left\{\frac{\stackrel{\star}{\Phi}}{\Phi} \stackrel{\star}{g}_{\alpha \beta}-\stackrel{\star \star}{g}_{\alpha \beta}+g^{\lambda \mu} \stackrel{\star}{g}_{\alpha \lambda} \stackrel{\star}{g}_{\beta \mu}-\frac{1}{2} g^{\mu \nu} \stackrel{\star}{g}_{\mu \nu} \stackrel{\star}{g}_{\alpha \beta}+\frac{1}{4} g_{\alpha \beta}\left[\stackrel{\star \mu \nu}{g} \stackrel{\star}{g}_{\mu \nu}+\left(g^{\mu \nu} \stackrel{\star}{g}_{\mu \nu}\right)^{2}\right]\right\},
$$

with the bars $(\|)$ denoting covariant derivative in a Riemannian sense and the star $(\star)$ denoting derivative with respect to the fifth coordinate $l$, and the function $\Lambda(x)$ is given by

$$
\Lambda(x)=\left.\frac{1}{4}(6-5 \xi) \Phi^{-2} \phi_{l}^{2}\right|_{l=l_{0}} .
$$

Clearly, both terms $T_{\alpha \beta}^{(I M)}$ and $\Lambda(x)$ comes from the Weylian bulk. The induced energy-momentum tensor $T_{\alpha \beta}^{(I M)} \mathrm{can}$ be obtained even if the bulk is Riemannian, but the interesting fact here is that the function $\Lambda(x)$ is a new contribution depending directly on the Weyl scalar field. It is worth mentioning that when the lapse function $\Phi$ dependends only on the time then $\Lambda(t)$ can be interpreted as an induced cosmological parameter, whereas if $\Phi$ is constant then 31 reduces to an induced cosmological constant.

\section{A SIMPLE APLiCATION TO COSMOLOGY}

As a simple application of the ideas developed in the previous section let us have a quick look into the cosmological scenario that takes place in the four-dimensional brane $\Sigma_{0}$, whose geometry is induced by the line element (20). In this case the induced line element (27) becomes

$$
d S_{\Sigma_{0}}^{2}=d t^{2}-a^{2}(t) d r^{2}
$$

which is nothing more than the line element of a Friedmann-Robetson-Walker model. The induced energy-momentum tensor (29) reduces to

$$
T_{\alpha \beta}^{(I M)}=F_{, \alpha, \beta}+F_{, \alpha} F_{, \beta}-{ }^{(4)}\left\{\begin{array}{l}
\gamma \\
\alpha \beta
\end{array}\right\} F_{, \gamma},
$$

where the ${ }^{(4)}\left\{\begin{array}{c}\gamma \\ \alpha \beta\end{array}\right\}$ denote the four-dimensional Christoffel symbols calculated with the induced metric $h_{\mu \nu}$ in (31). Assuming that the induced matter configuration given by (32) is that of a perfect fluid, as viewed by four-dimensional comoving observers located at the brane $\Sigma_{0}$, we can define the energy density $\rho_{(I M)}$ and pressure $P_{(I M)}$ for the induced matter by $\rho_{(I M)}=T^{(I M) t}{ }_{t}$ and $P_{(I M)}=-T^{(I M) r}{ }_{r}$ respectively. Thus using (31) the $4 \mathrm{D}$ field equations (28) becomes

$$
\begin{aligned}
3 H^{2} & =\rho_{(I M)}+\Lambda(t), \\
2 \frac{\ddot{a}}{a}+H^{2} & =-\left(P_{(I M)}-\Lambda(t)\right),
\end{aligned}
$$

where according to (30) and (21) the induced varying cosmological "constant" $\Lambda(t)$ is given by

$$
\Lambda(t)=\left(\frac{C_{1}}{2}\right)^{2}(6-5 \xi) e^{-2 F(t)} .
$$

Introducing the effective energy density $\rho_{\text {eff }}=\rho_{(I M)}+\Lambda(t)$ and the effective pressure $P_{\text {eff }}=P_{(I M)}-\Lambda(t)$ we define a parameter $\omega_{\text {eff }}$ associated with the effective equation of state, which is given by

$$
\omega_{e f f} \equiv \frac{P_{e f f}}{\rho_{e f f}}=-\left[1-\frac{\dot{F}^{2}+\ddot{F}-H \dot{F}}{\ddot{F}+\dot{F}^{2}+\Lambda(t)}\right]
$$


By simple inspection it can easily be seen that $\omega_{\text {eff }}$ depends entirely on the metric function $F(t)$, which, in turn, can be determined by the bulk dynamics, i.e. by finding solutions of the system (22)-(24). A particular solution $F(t)$ for a given scale factor $a(t)$ can be obtained by solving the equation (26). Thus if we look for solutions $F(t)$ in the case of a power-law expanding universe with the scale factor given by $a(t)=a_{0}\left(t / t_{0}\right)^{p}$, the equation (26) becomes

$$
\ddot{u}+\frac{4 p(2 p-1)}{t^{2}} u=0,
$$

whose general solution is given by

$$
u(t)=A_{1} t^{1 / 2+(1 / 2) \sqrt{1-32 p^{2}+16 p}}+A_{2} t^{1 / 2-(1 / 2) \sqrt{1-32 p^{2}+16 p}},
$$

where $A_{1}$ and $A_{2}$ are integration constants. Moreover, choosing $A_{2}=0$, the corresponding particular solution for $F(t)$ can be written as

$$
F(t)=\ln \left(B_{1} t^{\gamma}\right)
$$

where $B_{1}=\left(A_{1} t_{0}^{p} / a_{0}\right)$ and $\gamma=(1 / 2-p)+(1 / 2) \sqrt{1-32 p^{2}+16 p}$. Note that if we want to have real values for the power $\gamma$ that are compatible with an expanding universe $(p>0)$, the values of $p$ must range in the interval $0<p \leq(1 / 4)+\sqrt{6} / 8$. On the other hand, if we insert (39) into (35) and (36), then the induced variable cosmological "constant" $\Lambda(t)$ and the effective parameter $\omega_{\text {eff }}$ are given, respectively, by

$$
\begin{aligned}
\Lambda(t) & =\left(\frac{C_{1}}{2}\right)^{2}(6-5 \xi) B_{1}^{-2} t^{-2 \gamma} \\
\omega_{e f f} & =-\left[1-\frac{\gamma^{2}-\gamma-p \gamma}{\gamma^{2}-\gamma+\left(C_{1} / 2\right)^{2}(6-5 \xi) B_{1}^{-2} t^{2-2 \gamma}}\right] .
\end{aligned}
$$

If we want to have $\omega_{\text {eff }}$ decreasing with time we must require $2-2 \gamma>0$, and this condition restricts the range of variation of the parameter $p$ to $p>1 / 3$. Finally, if the former inequality is to be compatible with an expanding universe $p$ must range in the interval $1 / 3<p \leq(1 / 4)+\sqrt{6} / 8$. One reason for restricting the parameter $p$ to this interval is that with a suitable choice of $p$ the effective parameter $\omega_{\text {eff }}$ will tend asymptotically to -1 . We conclude that, in its final state, our model would tend to a de Sitter universe. Finally, note that in the case when $p=5 / 9$ the induced $\Lambda(t)$ given by (40) becomes a constant, while the value of $\omega_{e f f}$ is exactly -1 . This means that, in such models, $p=5 / 9$ corresponds to a de Sitter universe.

\section{FINAL REMARKS}

In this paper we have considered the idea of generating a cosmological constant, or rather, a cosmological parameter, from extra dimensions. Although this has already been investigated in the context of induced matter theory, the novelty of our approach is to regard the same problem in a more general setting, i.e by assuming the geometry of the embedding space to have a Weylian character. Two comments are in order: Firstly, the embedding space has a prescribed dynamics; secondly, the embedding does not affect the Riemannian geometry of the spacetime. These features depend on the fact that theWeyl field is assumed to be integrable and depending only on the extra dimension. Finally, by setting up a simple "toy model " our intention is to call attention to the richness of nonRiemannian geometries, in particular to the Weyl integrable manifolds, as a way of providing new degrees of freedom that might play a role in the theoretical framework of higher-dimensional embedding theories of spacetime. We believe that in this context issues such as the nature of the cosmological constant, dark energy and other important questions may be investigated from an entirely new point of view.

\section{Acknowledgements}

The authors would like to thank CNPq-CLAF and CNPq-FAPESQ (PRONEX) for financial support. We are indebted to Dr. F. Dahia for helpful comments.

[1] A. Einstein, A., "Zum kosmologischen Problem der allgemeinen Relativitätstheorie", Sitz. Ber. $\backslash$ Preuss. Akad. Wiss., 142, 235-237, (1931). 
[2] See, for instance, M. Tegmark et al.,Phys. Rev. D69 103501 (2004).

[3] S. Weinberg, Rev. Mod. Phys., 61, 1 (1989).

[4] See, for instance, H. F. M. Goenner, "On the History of Unified Field Theories", Living Rev. Rel., 7, 2 (2004)

[5] N. Arkani-Hamed, S. Dimopoulos, and G. Dvali, Phys. Lett. B 429, 263 (1998); I. Antoniadis, N. Arkani-Hamed, S. Dimopoulos, and G. Dvali, Phys. Lett. B 436, 257 (1998). L. Randall and R. Sundrum, Phys. Rev. Lett. 83, 3370 (1999); L. Randall and R. Sundrum, Phys. Rev. Lett. 83, 4690 (1999).

[6] P.S. Wesson and J. Ponce de Leon, J. Math. Phys. 33, 3883 (1992); J.M. Overduin and P.S. Wesson, Phys. Rept. 283 ,303 (1997), gr-qc/9805018 P.S. Wesson, Space-Time-Matter, (World Scientific, Singapore 1999); P. S. Wesson, FiveDimensional Physics (World Scientific, 2006).

[7] J. E. Campbell, A Course of Differential Geometry, Clarendon, 1926. L. Magaard, Zur Einbettung Riemannscher Raume in Einstein-raume und konform-euclidische Raume, PhD Thesis, Kiel, 1963

[8] C. Romero, R. Tavakol, and R. Zalaletdinov, Gen. Rel. Grav. 28, 365 (1995). F. Dahia and C. Romero, J. Math. Phys. 43, 5804 (2002). E. Anderson and J. E. Lidsey, Class. Quant. Grav.18, 4831 (2001). F. Dahia and C. Romero, J. Math. Phys. 43, 3097 (2002). E. Anderson, F. Dahia, James E. Lidsey, C. Romero, J. Math.Phys. 44, 5108 (2003). F. Dahia and C. Romero, Class. Quant. Grav. 21, 927 (2004). F. Dahia and C. Romero, Class. Quant. Grav. 22, 5005 (2005)

[9] T. Kaluza, Sitz. Preuss. Akad. Wiss. 33, 966 (1921). O. Klein, Z. Phys. 37, 895 (1926). T. Appelquist, A. Chodos and P. Freund, Modern Kaluza-Klein Theories, Addison-Wesley, Menlo Park, 1987.

[10] P. S. Wesson and J. Ponce de Leon, J.Math.Phys.33, 3883 (1992).

[11] Maia, M. D., Hypersurfaces of five-dimensional vacuum space-times, gr-qc/9512002.

[12] J. Ponce de Leon, Gen. Rel. Grav. 20, 539.

[13] H. Weyl, Sitzungesber Deutsch. Akad. Wiss. Berlin, 465 (1918). H. Weyl, Space, Time, Matter (Dover, New York, 1952)

[14] See, for instance, W. Pauli, Theory of Relativity (Dover, New York, 1981). See, also, L. O'Raiefeartaigh and N. Straumann, Rev. Mod. Phys. 72, 1 (2000).

[15] F. Dahia, G. A. T. Gomez and C. Romero, [ArXiv:gr-qc/0711.2754].

[16] M. Novello, L.A.R. Oliveira, J.M. Salim, E. Elbas, Int. J. Mod. Phys. D1 (1993) 641-677. J. M. Salim and S. L. Sautú, Class. Quant. Grav. 13, 353 (1996). H. P. de Oliveira, J. M. Salim and S. L. Sautú, Class.Quant.Grav. 14, 2833 (1997). V. Melnikov, Classical Solutions in Multidimensional Cosmology in Proceedings of the VIII Brazilian School of Cosmology and Gravitation II (1995), edited by M. Novello (Editions Frontières) pp. 542-560, ISBN 2-86332-192-7.

[17] Mark Israelit, Found. Phy. Vol. $35 \mathrm{~N}^{\circ} 10$ (2005) 1725-1748.

[18] F. Dahia, L. F. P. da Silva, C. Romero e R. Tavakol, J. Math. Phys. 48, 072501 (2007). F. Dahia, L. F. P. da Silva, C. Romero e R. Tavakol, Gen. Rel. Grav. 40, 1341 (2008).

[19] See, for instance, C. W. Misner, K. S. Thorne and J. A. Wheeler, Gravitation, Ch. 21 (Freeman, 1973) 